\title{
HIV-positive women with anal high-grade squamous intraepithelial lesions: a study of 153 cases with long-term anogenital surveillance
}

\author{
Yuxin Liu $\oplus^{1} \cdot$ Monica Prasad-Hayes ${ }^{2} \cdot$ Eric M. Ganz ${ }^{2}$ Juan Lucas Poggio ${ }^{3}$. Volha Lenskaya ${ }^{1} \cdot$ Threshia Malcolm $^{3}$. \\ Ashish Deshmukh ${ }^{4} \cdot$ Wenxin Zheng ${ }^{5} \cdot$ Keith Sigel $^{6} \cdot$ Michael M. Gaisa (iD $^{7}$
}

Received: 8 January 2020 / Revised: 25 February 2020 / Accepted: 26 February 2020 / Published online: 9 March 2020

(c) The Author(s), under exclusive licence to United States \& Canadian Academy of Pathology 2020

\begin{abstract}
Women living with HIV (WLHIV) are at increased risk for human papillomavirus (HPV)-associated anal cancer. Given the "field effect" of HPV pathogenesis, some recommend that anal cancer screening should be limited to WLHIV with prior genital disease. This study aimed to characterize the relationship between anal and genital disease in WLHIV in order to better inform anal cancer screening guidelines. We retrospectively studied 153 WLHIV with biopsy-proven anal high-grade squamous intraepithelial lesions (AHSIL) and long-term evaluable cervical/vaginal/vulvar histopathology. Based on the absence or presence of genital HSIL, subjects were categorized as having isolated AHSIL or multicentric HSIL. Demographics, HIV parameters and cervical/anal HPV status were recorded. Chi-square test was used for bivariate analyses. Of 153 WLHIV with AHSIL, 110 (72\%) had isolated AHSIL, while 43 (28\%) had multicentric HSIL (28 cervical, 16 vulvar, and 8 vaginal HSIL). The median genital surveillance was 8 years (range 1-27). Cervical HPV16/18 infection was associated with multicentric disease $(P=0.001)$. Overall, 53\% of multicentric cases presented genital HSIL preceding AHSIL with median interval 13 years (range 2-23). Paired anal and cervical high-risk HPV results were available for 60 women within 12 months of AHSIL diagnosis: 30 (50\%) had anal infection alone, while $30(50 \%)$ had anal/cervical coinfection by 16/18 (15\%), non-16/18 (13\%), or different types (22\%). In conclusion, WLHIV frequently develop AHSILs without pre-existing genital disease or after long latency following a genital HSIL diagnosis. Our findings support anal cancer screening for WLHIV irrespective of prior genital disease.
\end{abstract}

Yuxin Liu

Yuxin.liu@mountsinai.org

1 Department of Pathology, Icahn School of Medicine at Mount Sinai, New York, NY, USA

2 Department of Obstetrics, Gynecology and Reproductive Science, Icahn School of Medicine at Mount Sinai, New York, NY, USA

3 Division of Colorectal Surgery, Department of surgery, Drexel University College of Medicine, Philadelphia, PA, USA

4 Center for Health Services Research, Department of Management, Policy, and Community Health, UT Health School of Public Health, Houston, TX, USA

5 Department of Pathology, Obstetrics and Gynecology, Simon Comprehensive Cancer Center, University of Texas Southwestern Medical Center, Dallas, TX, USA

6 Division of General Internal Medicine, Department of Medicine, Icahn School of Medicine at Mount Sinai, New York, NY, USA

7 Division of Infectious Diseases, Department of Medicine, Icahn School of Medicine at Mount Sinai, New York, NY, USA

\section{Introduction}

In United States, the incidence of human papillomavirus (HPV)-associated anal cancer has risen by $150 \%$ among women between 1999 and 2015 [1]. The American Cancer Society projects $\sim 8300$ new anal cancer cases in 2019 [2]. Two-thirds of these cases will affect women and nearly half will present at advanced stages, underscoring a serious deficiency in anal cancer early detection [3]. Women living with the human immunodeficiency virus (WLHIV) and/or lower genital tract neoplasia (LGTN) are at increased risk for anal HPV infection and anal cancer [4-7]. HIV infection impairs host immunity, creating a favorable environment for HPV to persist and progress [8, 9]. LGTN reflects a pervasive "field effect" in HPV pathogenesis wherein infection at one genital site causes sequentially higher risk of HPV exposure leading to dysplasia or malignancy at other genital sites [10].

Anal high-grade squamous intraepithelial lesions (AHSIL) are the immediate cancer precursors and therefore 
primary targets for preventive screening and treatment [11]. Recently, the AIDS Malignancy Consortium 084 study reported an AHSIL prevalence of $27 \%$ in WLHIV in the US [12]. Unlike the well-established cervical cancer screening algorithms, there are no universally accepted anal cancer screening guidelines for WLHIV [3]. Several organizations advocate for screening selective groups; for example, the AIDS Institute of the New York State Department of Health and American Cancer Society recommend annual anal cytology for WLHIV with a history of abnormal cervical/ vulvar histology [13], (https://www.cancer.org/cancer/analcancer/detection-diagnosis-staging/detection.html). In addition, the HIV Medicine Association of the Infectious Diseases Society of America promotes screening of WLHIV with a history of receptive anal intercourse (RAI) [14]. Currently, there are no screening recommendations for women with syphilis or other sexually transmitted infections. In 2015, drawing upon expert opinions, the American Society for Colposcopy and Cervical Pathology (ASCCP) endorsed the most comprehensive recommendation of screening all WLHIV for anal cancer [3]. It is yet to be determined whether this universal screening strategy will become standard of care, and if so, how it will be implemented in clinical practice.

Herein, we set out to characterize a cohort of WLHIV with biopsy-proven AHSIL with a goal to determine the relationship between AHSIL and cervical/vaginal/vulvar HSIL by evaluating lesion distribution, chronological sequence and HPV genotypes. Our findings provide direct evidence whether genital abnormalities alone could serve as a criterion for anal screening eligibility, which should inform anal cancer screening guidelines.

\section{Materials and methods}

\section{Case selection and anogenital surveillance}

The Institutional Review Boards of the Icahn School of Medicine at Mount Sinai approved this study. We searched clinical anal dysplasia databases from January 2010 to January 2018 for WLHIV with high-resolution anoscopy (HRA)-guided biopsy-proven intra-anal HSIL. Only women with cervical/vaginal/vulvar surveillance and histopathological examination were included in the study.

Medical charts were reviewed for demographics (age, race/ethnicity), HIV status, history of smoking, RAI and CD4 count (categorized as $<200$ cells $/ \mathrm{mm}^{3}, 200-500$ cells/ $\mathrm{mm}^{3}$ and $>500$ cells $/ \mathrm{mm}^{3}$ ) and HIV RNA level (categorized as $<100$ copies/ml versus $>100$ copies/ml) within 12 months of AHSIL diagnosis. Cervical/vaginal/vulvar histological diagnoses were extracted from pathology database including biopsy, endocervical curettage, loop electrosurgical excision procedure (LEEP), conization, vulvectomy, and hysterectomy. Genital surveillance time referred to the interval between initial and most recent gynecological evaluation. Number of genital examinations referred to the total number of cervical cytology and surgical procedures performed during the surveillance period.

Participants were classified as having isolated AHSIL when no cervical/vaginal/vulvar HSILs were detected by any gynecological examination, cytology, biopsy, or excision. When such lesions were detected in addition to AHSIL, participants were classified as having multicentric HSIL.

\section{Anal cancer screening}

As a distinct institutional policy, beginning in 2009, all WLHIV at the Icahn School of Medicine have been offered anal cancer screening with anal cytology by gynecologists or infectious disease specialists [15]. Those with abnormal anal cytology results (Atypical Squamous Cells of Undetermined Significance or higher grade abnormalities) are referred for HRA. HRA procedures were performed in an office setting following previously described techniques [16]. Briefly, the perianal area, distal anal canal, and squamocolumnar junction were treated with $3 \%$ acetic acid and Lugol's iodine and then visualized under a highresolution colposcope at 15 -fold magnification. Any areas with abnormal mucosal appearance suspicious for dysplasia or cancer were biopsied.

Surgical pathologists at the Mount Sinai Hospital diagnosed all anal biopsies on hematoxylin and eosin slides following Lower Anogenital Squamous Terminology criteria [17]. Two pathologists (YL and WZ) independently reviewed and confirmed the diagnoses of AHSIL for all cases included in the study. Both authors are specialized gynecological pathologists with 10 and 30 years of experience in diagnosing HPV-related anogenital disease. The designation of AHSIL required nuclear enlargement, coarse chromatin, irregular nuclear membrane and mitoses involving two-third or full-thickness of the squamous epithelium.

\section{Anal and cervical HPV genotyping}

Both anal and cervical high-risk HPV results were limited to tests performed within 12 months of AHSIL diagnosis. HPV genotyping was performed on cervical or anal cytology fluid using the Roche Cobas ${ }^{\circledR} 4800$ HPV assay (Roche Diagnostics, Indianapolis, IN) capable of identifying HPV16, 18, and 12 other high-risk types $(31,33,35,39,45$, $51,52,56,58,59,66$, and 68 ). Results were recorded as HPV16/18 when HPV16 and/or 18 were detected with or without other types. Results were recorded as non-16/18 
types when any of the 12 other high-risk types were detected. If none of the 14 types was detected, HPV status was recorded as negative.

\section{Statistical analysis}

We compared demographic and clinicopathological characteristics of patients with isolated AHSIL versus those with multicentric HSIL using the Wilcoxon test for continuous variables (age, follow-up and number of examinations) and the chi-square test for categorical variables. All analyses were performed in STATA Version 15 (STATA Corporation, College Station, Texas).

\section{Results}

\section{Isolated AHSIL versus multicentric HSIL}

A total of 153 WLHIV with biopsy-proven AHSIL were included in the study. Demographic and clinicopathological are shown in Table 1. All participants were prescribed antiretroviral therapy during the study period. Biopsies of cervix/vagina/vulva revealed benign mucosa or low-grade squamous intraepithelial lesions (LSIL) in 110 (72\%) women (isolated AHSIL). Genital HSIL was detected in 43 (28\%) women (multicentric HSIL), including 28 cervical HSIL, 16 vulvar HSIL, and 8 vaginal HSIL. Nine women harbored HSIL at three or four genital sites. During followup, four women progressed to invasive cervical or vulvar squamous cell carcinoma. None progressed to invasive anal squamous cell carcinoma.

Comparing isolated AHSIL and multicentric HSIL groups (Table 1), cervical HPV16/18 positivity was associated with multicentric disease $(P=0.002)$. There was no significant difference in other demographic variables such as age, race/ethnicity, smoking, RAI, HIV parameters, or anal HPV types.

\section{Chronological sequence of anal and genital HSIL}

As shown in Table 2, based on the time of histopathological diagnosis, multicentric cases were categorized into three groups: (1) Genital HSIL preceded AHSIL $(n=23,53 \%)$ with a median interval of 13 years (range 2-23). The median genital surveillance was 19 years (range 6-27). All participants were treated with one or more LEEP procedures for cervical HSIL, resections for vulvar/vaginal HSIL, and five underwent hysterectomy. (2) AHSIL preceded genital HSIL $(n=3,7 \%)$ with a median interval of 2 years (range 2-3). The genital surveillance was 5, 6, and 24 years for these three patients, respectively. (3) AHSIL and genital HSIL diagnosed within a 12-month time period $(n=17$,
Table 1 A comparison of characteristics between women with isolated AHSIL and those with multicentric HSIL.

\begin{tabular}{|c|c|c|c|c|}
\hline Characteristics & $\begin{array}{l}\text { Total } \\
(N=153)\end{array}$ & $\begin{array}{l}\text { Isolated } \\
\text { AHSIL } \\
(N=110)\end{array}$ & $\begin{array}{l}\text { Multicentric } \\
\operatorname{HSIL}(N=43)\end{array}$ & $P$ value $^{\mathrm{a}}$ \\
\hline $\begin{array}{l}\text { Age at initial AHSIL } \\
\text { (years) }\end{array}$ & $50(20-87)$ & $51(26-87)$ & $49(20-67)$ & 0.3 \\
\hline $\begin{array}{l}\text { Length of genital } \\
\text { surveillance (years) }\end{array}$ & $8(1-27)$ & $8(1-24)$ & $16(1-27)$ & $<0.001$ \\
\hline $\begin{array}{l}\text { Number of genital } \\
\text { examination }\end{array}$ & $8(1-40)$ & $7(1-40)$ & $16(2-40)$ & $<0.001$ \\
\hline \multicolumn{5}{|l|}{ Race/ethnicity } \\
\hline African American & $69(45)$ & $44(40)$ & $25(58)$ & \multirow[t]{5}{*}{0.1} \\
\hline White & $8(5)$ & $7(6)$ & $1(2)$ & \\
\hline Hispanic & $44(29)$ & $36(25)$ & $8(19)$ & \\
\hline Other & $17(11)$ & $12(8)$ & $5(12)$ & \\
\hline Unknown & $15(10)$ & $11(10)$ & $4(9)$ & \\
\hline \multicolumn{5}{|l|}{ Smoking status } \\
\hline Current & $53(35)$ & $41(37)$ & $12(28)$ & \multirow[t]{4}{*}{0.5} \\
\hline Former & $35(23)$ & $24(22)$ & $11(26)$ & \\
\hline Never & $32(21)$ & $21(19)$ & $11(26)$ & \\
\hline Unknown & $33(21)$ & $24(22)$ & $9(17)$ & \\
\hline \multicolumn{5}{|c|}{ Receptive anal intercourse } \\
\hline Yes & $101(66)$ & $74(67)$ & $27(63)$ & \multirow[t]{3}{*}{0.5} \\
\hline No & $30(20)$ & $24(22)$ & $6(14)$ & \\
\hline Unknown & $22(14)$ & $12(11)$ & $10(23)$ & \\
\hline \multicolumn{5}{|c|}{ HIV RNA load, copies/mL } \\
\hline$<100$ & 117 (77) & $84(76)$ & $33(77)$ & \multirow[t]{3}{*}{0.8} \\
\hline$\geq 100$ & $34(22)$ & $25(23)$ & $9(21)$ & \\
\hline Unknown & $2(1)$ & $1(1)$ & $1(2)$ & \\
\hline \multicolumn{5}{|c|}{ CD4+ T-cell count, cells $/ \mathrm{mm}^{3}$} \\
\hline$<200$ & $25(16)$ & $15(14)$ & $10(23)$ & \multirow[t]{4}{*}{0.3} \\
\hline $200-500$ & $47(31)$ & $34(31)$ & $13(30)$ & \\
\hline$>500$ & $79(52)$ & $60(54)$ & $19(44)$ & \\
\hline Unknown & $2(1)$ & $1(1)$ & $1(2)$ & \\
\hline \multicolumn{5}{|c|}{ Number of anal high-grade lesions } \\
\hline 1 or 2 & $126(82)$ & $87(79)$ & $39(91)$ & \multirow[t]{2}{*}{0.09} \\
\hline $3-6$ & $27(18)$ & $23(21)$ & $4(9)$ & \\
\hline \multicolumn{5}{|c|}{ Cervical HPV genotype within 12 months AHSIL } \\
\hline $16 / 18$ & $17(19)$ & $6(10)$ & $11(40)$ & \multirow[t]{4}{*}{0.002} \\
\hline Non- $16 / 18^{\mathrm{b}}$ & $29(33)$ & $21(34)$ & $8(30)$ & \\
\hline Negative & $42(48)$ & $34(56)$ & $8(30)$ & \\
\hline Total number & 88 & 61 & 27 & \\
\hline \multicolumn{5}{|c|}{ Anal HPV genotype within 12 months AHSIL } \\
\hline $16 / 18$ & $51(70)$ & $41(73)$ & $10(59)$ & \multirow[t]{3}{*}{0.2} \\
\hline Non- $16 / 18^{b}$ & $22(30)$ & $15(27)$ & $7(41)$ & \\
\hline Total number & 73 & 56 & 17 & \\
\hline
\end{tabular}

$\overline{\text { Data are presented as median (range) or number of cases (percentage). }}$ ${ }^{\mathrm{a} C}$ Comparison of multicentric versus isolated HSIL.

bNon-16/18: HPV 31, 33, 35, 39, 45, 51, 52, 56, 58, 59, 66, and 68.

$40 \%$ ). The median genital surveillance for this group was 8 years (range 1-26).

\section{Anal and cervical high-risk HPV genotypes}

Within 12 months of AHSIL diagnosis, 52\% (46/88) tested positive for cervical high-risk HPV, while all (73/73) 
Table 2 Chronological sequence of genital HSIL and AHSIL diagnosis in women with multicentric HSIL $(n=43)$.

\begin{tabular}{lllcc}
\hline Group & Chronological sequence & Interval between genital and anal HSIL & Number of case (\%) & Length of surveillance \\
\hline 1 & Genital HSIL preceded AHSIL & 13 years (2-23) & $23(53 \%)$ & 19 years $(6-27)$ \\
2 & AHSIL preceded genital HSIL & 2 years (2-3) & $3(7 \%)$ & 6 years (5-24) \\
3 & Synchronous & Less than one year & $17(40 \%)$ & 8 years (1-26) \\
\hline
\end{tabular}

Data are given as median (range).

Table 3 HPV distribution in women with concurrent anal and cervical HPV genotyping $(n=60)$.

\begin{tabular}{lllr}
\hline HPV distribution & $\begin{array}{l}\text { Anal } \\
\text { HR-HPV }\end{array}$ & $\begin{array}{l}\text { Cervical } \\
\text { HR-HPV }\end{array}$ & $N(\%)$ \\
\hline Anal infection alone & $16 / 18^{\mathrm{a}}$ & - & $20(33)$ \\
$(n=30,50 \%)$ & Non-16/18 & - & $10(17)$ \\
Anal/cervical coinfection & $16 / 18$ & $16 / 18$ & $9(15)$ \\
$(n=30,50 \%)$ & $16 / 18$ & Non-16/18 & $13(22)$ \\
& Non-16/18 & Non-16/18 & $8(13)$ \\
Total & & & $60(100)$
\end{tabular}

${ }^{\mathrm{a}} 16 / 18$ includes the detection of HPV16 and/or 18 with or without other high-risk types.

${ }^{\mathrm{b}}$ Non-16/18: HPV 31, 33, 35, 39, 45, 51, 52, 56, 58, 59, 66, and 68.

women tested positive for anal high-risk HPV (Table 1). Paired anal and cervical HPV results were available for 60 women (Table 3). Of them, 30 had anal infection alone, while $30 \mathrm{had}$ anal/cervical coinfection in the following combinations: $16 / 18$ at both sites $(n=9,15 \%)$, anal $16 / 18$ combined with cervical non-16/18 $(n=13,22 \%)$, and non$16 / 18$ types at both sites $(n=8,13 \%)$.

\section{Discussion}

In this retrospective study, we found that among WLHIV, anal precancer frequently manifests as an isolated lesion or after considerable intervals following other HPV-associated genital precancers. This observation underscores the importance of anal cancer screening for WLHIV with a history of genital disease, but also makes a strong case for screening women without such history. Rather than making anal cancer screening contingent upon pre-existing genital disease, we advocate for expanding screening to all WLHIV.

Despite the high risk of LGTN, overall $72 \%$ of our subjects were found to have cancer precursors limited to the anal canal. Long-term and frequent gynecological surveillance did not detect any severe cervical, vaginal or vulvar dysplasia. These women would not have been eligible for anal screening if genital HSIL had been set as the referral standard. In our previous study of 745 WLHIV, the use of cervical/vulvar abnormality as a criterion would have excluded $21 \%$ of AHSIL patients from screening [15]. Similarly, a publication based on the Women's Interagency HIV Study (WIHS) reported that the severity of anal lesions correlated poorly with cervical lesions [18]. In their study, only 4 out of 30 (13\%) WLHIV harbored HSIL concurrently at both genital sites, while $26(87 \%)$ had AHSIL combined with benign cervical findings or LSIL. Recently, Stier et al. found that AHSIL prevalence rates of $25 \%$ and $28 \%$ in WLHIV who had concomitant benign or ASCUS/ LSIL cervical cytology, respectively [12]. These studies, in accordance with our findings suggest that absent or mild genital abnormalities do not preclude more advanced neoplastic disease in the anal canal. Considering the strong synergy between HIV and HPV, these data support anal cancer screening for all WLHIV, even those without significant genital abnormalities.

Due to proximity, HPV tends to spread from one genital site to another, inducing multicentric lesions synchronously or metachronously, a phenomenon known as the field effect [19]. Multicentric HSIL, however, accounted only for $28 \%$ of our cases, predominately involving cervix, vulva, and in rare cases, vagina. Notably, the most common presentation we observed was genital HSIL preceding AHSIL by a prolonged latency (median 13 years). Despite differences in population and outcome, similar chronological sequences and intervals between cervical precancer and anal cancer have been noted in several population-based studies. Using data from the England Thames Cancer Registry, Evans et al. reported that the incidence of anal cancer increased significantly 10 or more years following cervical HSIL [20]. A US SEER-based study observed an even longer interval of 16 years from cervical HSIL to anal cancer [21]. While ASCCP recommends anal screening within 5 years of cervical/vaginal precancer or cancer diagnosis, our findings suggest that anal surveillance be continued at least one decade, or even indefinitely in the case of WLHIV, in order to capture any delayed malignant transformation in the anal canal.

The HSIL distribution we observed, whether isolated or multicentric, showed variable correlation with individual patients' cervical high-risk HPV status. Women with isolated AHSIL tended to be negative for cervical high-risk HPV (56\%) or positive for non-16/18 types (34\%). 
Conversely, $70 \%$ of multicentric cases were positive for cervical high-risk HPV, predominately HPV16/18 (40\%). A recent meta-analysis of anal and cervical HPV prevalence found high correlation between the two genital sites [22]. In their analysis, AHSIL was associated with cervical high-risk HPV (particularly HPV16); AHSIL prevalence was low $(8 \%)$ in women with negative cervical HPV. Our multicentric cases are in line with their findings, but isolated AHSIL cases diverge, as $56 \%$ of them were negative for cervical HR-HPV within 12 months of AHSIL diagnosis. This discrepancy raises the question as to whether the host's immune response clears cervical and anal HPV by a different mechanism or with different velocities. The anal canal may provide a more favorable microenvironment for HPV to escape host immune surveillance and establish persistent, transforming infection [5].

In analyses of women with paired anal and cervical HPV testing within 12 months of AHSIL diagnosis, we found that high-risk HPV was more prevalent in the anus than cervix (100\% vs. 52\%), particularly HPV16/18 (70\% vs. $19 \%)$. Similar HPV distributions were observed in the SUN study wherein the prevalence of anal and cervical high-risk HPV was 85 and $70 \%$ in WLHIV at baseline [23]. The WIHS study (primarily comprising WLHIV with benign anal/cervical findings) reported that HPV16 and 18 were threefold and fourfold more prevalent in the anus compared with cervix [18]. Differences in patient cohorts notwithstanding, these findings further support the possibility of differential responses to HPV infection at these two genital sites and suggest a higher risk of anal than cervical disease for WLHIV.

Our study has several strengths: long-term gynecological surveillance, HRA-guided anal biopsy with histological diagnosis, and paired cervical/anal HPV genotyping. One limitation is the wide variation among subjects regarding the timing and frequency of gynecological and anal examinations. While two-thirds of our cohort was regularly followed every 6 months or annually, the remaining subjects had only sporadic examination every 2 or 3 years.

In summary, HPV-associated anal cancer precursors frequently present as isolated lesions in WLHIV or after considerable intervals following the treatment of genital HSIL. To prevent anal cancer in this high-risk population, our findings support anal cancer screening for WLHIV irrespective of prior genital disease.

Funding This work was supported by the National Cancer Institute (grant no. K07CA180782 to KS).

\section{Compliance with ethical standards}

Conflict of interest The authors declare that they have no conflict of interest.
Publisher's note Springer Nature remains neutral with regard to jurisdictional claims in published maps and institutional affiliations.

\section{References}

1. Van Dyne EA, Henley SJ, Saraiya M, Thomas CC, Markowitz LE, Benard VB. Trends in human papillomavirus-associated cancers-United States, 1999-2015. MMWR Morb Mortal Wkly Rep. 2018;67:918-24.

2. American Cancer Society. Atlanta G. Cancer Facts \& Figures 2019. Available at: https://www.cancer.org/cancer/anal-cancer/a bout/what-is-key-statistics.html.

3. Moscicki AB, Darragh TM, Berry-Lawhorn JM, Roberts JM, Khan MJ, Boardman LA, et al. Screening for anal cancer in women. J Low Genit Trac Dis. 2015;19:S27-42.

4. Ferenczy A, Coutlee F, Franco E, Hankins C. Human papillomavirus and HIV coinfection and the risk of neoplasias of the lower genital tract: a review of recent developments. CMAJ. 2003;169:431-4.

5. Stier EA, Sebring MC, Mendez AE, Ba FS, Trimble DD, Chiao EY. Prevalence of anal human papillomavirus infection and anal HPV-related disorders in women: a systematic review. Am J Obstet Gynecol. 2015;213:278-309.

6. Suk R, Mahale P, Sonawane K, Sikora AG, Chhatwal J, Schmeler $\mathrm{KM}$, et al. Trends in risks for second primary cancers associated with index human papillomavirus-associated cancers. JAMA Netw Open. 2018;1:e181999.

7. Edgren G, Sparen P. Risk of anogenital cancer after diagnosis of cervical intraepithelial neoplasia: a prospective population-based study. Lancet Oncol. 2007;8:311-6.

8. Liu Y, Gaisa MM, Wang X, Swartz TH, Arens Y, Dresser KA, et al. Differences in the Immune microenvironment of anal cancer precursors by HIV status and association with ablation outcomes. J Infect Dis. 2018;217:703-9.

9. Looker KJ, Ronn MM, Brock PM, Brisson M, Drolet M, Mayaud $\mathrm{P}$, et al. Evidence of synergistic relationships between HIV and Human Papillomavirus (HPV): systematic reviews and metaanalyses of longitudinal studies of HPV acquisition and clearance by HIV status, and of HIV acquisition by HPV status. J Int AIDS Soc. 2018;21:e25110.

10. Vinokurova S, Wentzensen N, Einenkel J, Klaes R, Ziegert C, Melsheimer $\mathrm{P}$, et al. Clonal history of papillomavirus-induced dysplasia in the female lower genital tract. J Natl Cancer Inst. 2005;97:1816-21.

11. Goldie SJ, Kuntz KM, Weinstein MC, Freedberg KA, Welton ML, Palefsky JM. The clinical effectiveness and costeffectiveness of screening for anal squamous intraepithelial lesions in homosexual and bisexual HIV-positive men. JAMA 1999;281:1822-9.

12. Stier EA, Lensing SY, Darragh TM, Deshmukh AA, Einstein MH, Palefsky JM, et al. Prevalence of and risk factors for anal highgrade squamous intraepithelial lesions in women living with human immunodeficiency virus. Clin Infect Dis. 2019. https://doi. org/10.1093/cid/ciz408. Epub ahead of print.

13. New York State Department of Health AIDS Institute. Anal dysplasia and cancer, 2007. Available at: http://www. hivguidelines.org/clinicalguidelines/adults/anal-dysplasia-and-ca ncer/.

14. Aberg JA, Gallant JE, Ghanem KG, Emmanuel P, Zingman BS, Horberg MA. Primary care guidelines for the management of persons infected with HIV: 2013 update by the HIV medicine association of the infectious diseases society of America. Clin Infect Dis. 2014;58:e1-34. 
15. Jay N, Berry JM, Hogeboom CJ, Holly EA, Darragh TM, Palefsky JM. Colposcopic appearance of anal squamous intraepithelial lesions: relationship to histopathology. Dis Colon Rectum. 1997;40:919-28.

16. Darragh TM, Colgan TJ, Thomas Cox J, Heller DS, Henry MR, Luff RD, et al. The lower anogenital squamous terminology standardization project for HPV-associated lesions: background and consensus recommendations from the College of American Pathologists and the American Society for Colposcopy and Cervical Pathology. Int J Gynecol Pathol. 2013;32:76-115.

17. Gaisa M, Ita-Nagy F, Sigel K, Arens Y, Hennessy MA, Rodriguez-Caprio G, et al. High rates of anal high-grade squamous intraepithelial lesions in HIV-infected women who do not meet screening guidelines. Clin Infect Dis. 2017;64:289-94.

18. Hessol NA, Holly EA, Efird JT, Minkoff H, Weber KM, Darragh TM, et al. Concomitant anal and cervical human papillomavirus infections and intraepithelial neoplasia in HIV-infected and uninfected women. AIDS. 2013;27:1743-51.
19. Adams TS, Mbatani NH. Clinical management of women presenting with field effect of HPV and intraepithelial disease. Best Pr Res Clin Obstet Gynaecol. 2018;47:86-94.

20. Evans HS, Newnham A, Hodgson SV, Møller H. Second primary cancers after cervical intraepithelial neoplasia III and invasive cervical cancer in Southeast England. Gynecol Oncol 2003;90:131-6.

21. Saleem AM, Paulus JK, Shapter AP, Baxter NN, Roberts PL, Ricciardi R. Risk of anal cancer in a cohort with human papillomavirus-related gynecologic neoplasm. Obstet Gynecol 2011;117:643-9.

22. Lin C, Slama J, Gonzalez P, Goodman MT, Xia N, Kreimer AR, et al. Cervical determinants of anal HPV infection and high-grade anal lesions in women: a collaborative pooled analysis. Lancet Infect Dis. 2019;19:880-91.

23. Kojic EM, Cu-Uvin S, Conley L, Bush T, Onyekwuluje J, Swan DC, et al. Human papillomavirus infection and cytologic abnormalities of the anus and cervix among HIV-infected women in the study to understand the natural history of HIV/AIDS in the era of effective therapy (the SUN study). Sex Transm Dis. 2011;38:253-9. 